\title{
Perinatal Pyogenic Liver Abscess: A Rare Entity and First Reported Case of Klebsiella pneumoniae
}

\author{
Stacey S. Schmiedecke, MD ${ }^{1}$ Peter G. Napolitano, MD ${ }^{1} \quad$ Sarah M. Estrada, MD \\ 1 Department of Obstetrics and Gynecology, Division of Maternal \\ Fetal Medicine, Madigan Army Medical Center, Tacoma, Washington \\ Am J Perinatol Rep 2019;9:e251-e255. \\ Address for correspondence Stacey S. Schmiedecke, MD, \\ Department of Obstetrics and Gynecology, Division of Maternal Fetal \\ Medicine, Madigan Army Medical Center, 9040 Jackson Avenue, \\ Tacoma, WA 98431 (e-mail: stacey.s.schmiedecke.mil@mail.mil).
}

\begin{abstract}
Keywords

- pyogenic liver abscess

- liver abscess

- pregnancy

- sepsis

- perinatal infection
\end{abstract}

Cryptogenic pyogenic liver abscess (PLA) describes those cases with unknown etiology and can be thought of as primary PLA rather than secondary to hepatobiliary disease, gastrointestinal infection, or intra-abdominal infection. Cryptogenic invasive PLA (CIPLA) describes the involvement of septic emboli to the brain, eyes, lungs, heart, joints, fascia, or other distant organs. Monomicrobial CIPLA secondary to Klebsiella pneumoniae (CIKPLA) is endemic to Taiwan with additional cases described in other Eastern countries, but just one case described in the United States and no prior reports in or around pregnancy. ${ }^{1}$ On the other hand, cryptogenic noninvasive PLA (CNPLA) is usually associated with bacteremia without septic seeding. Monomicrobial CNPLA secondary to K. pneumoniae, while rare, has been described in Western countries, though only in patients of Asian or Hispanic descent and never in the puerperium. To our knowledge, pregnancy-related cryptogenic PLA has not been previously described in the medical literature.

(D)Stacey S. Schmiedecke's ORCID is https://orcid.org/0000-00033577-2513.

received

April 14, 2019

accepted after revision

May 14, 2019
We report here a case of monomicrobial CNPLA in pregnancy secondary to K. pneumoniae in a patient of Asian descent with no other risk factors.

\section{Case}

A 29 year-old gravida 2 para 1001 of Filipino descent presented to the emergency department at 11 weeks and 1 day of gestation complaining of fever, myalgias, malaise, and headache. She reported no other symptoms and denied any sick contacts. Her medical history included childhood asthma with no current inhaler use. Her obstetric history was pertinent for an uncomplicated term spontaneous vaginal delivery three years prior. Her remaining history was unremarkable aside from reported allergy to penicillin. She had lived in the United States for 7 years and denied any recent travel.

On presentation, her vital signs were notable for blood pressure ranging $90-100 / 50-70 \mathrm{~mm} \mathrm{Hg}$, heart rate 100 to $120 \mathrm{bpm}$, oxygen saturation $93 \%$ to $100 \%$ on room air, and initial temperature of $38.7^{\circ} \mathrm{C}$ with maximum temperature of $39.6^{\circ} \mathrm{C}$. Physical examination was unremarkable and without

Copyright $\odot 2019$ by Thieme Medical Publishers, Inc., 333 Seventh Avenue, New York, NY 10001, USA.

License terms 10.1055/s-0039-1692713. ISSN 2157-6998. 


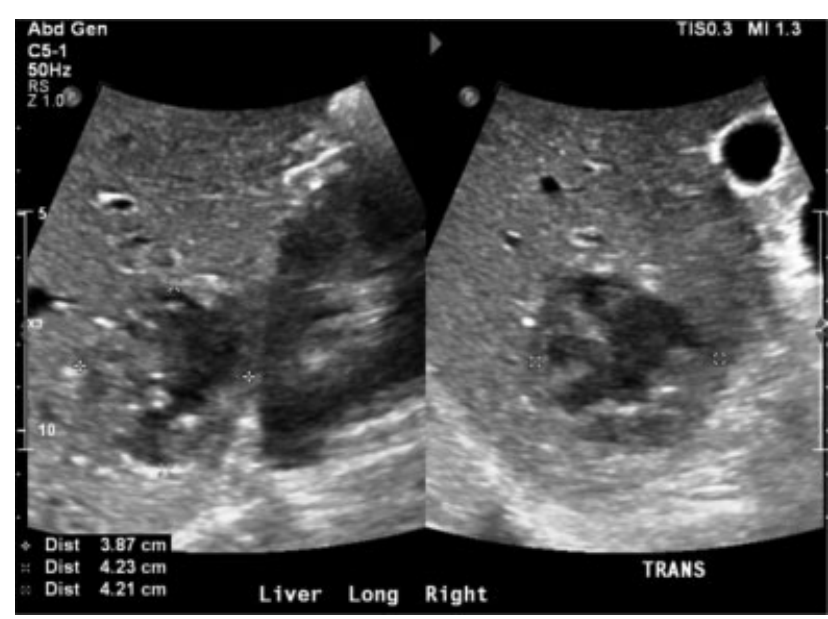

Fig. 1 Our patient's right upper-quadrant (RUQ) ultrasound demonstrated a $3.9 \times 4.2 \times 4.2 \mathrm{~cm}$ echogenic and irregular lesion within the right lobe of the liver adjacent to the right kidney. The partially ill-defined peripheral rim and absence of internal blood flow were suspicious for an early pyogenic abscess, and less likely a hypovascular tumor.

meningeal signs. Fetal heart rate was assessed by abdominal ultrasound at 160 to $170 \mathrm{bpm}$. Initial laboratory analysis was unremarkable including absence of leukocytosis (WBC 9.4), as well as normal renal and hepatic function. Urinalysis was also normal. Chest X-ray was without evidence of acute cardiopulmonary process. Blood and urine cultures were obtained. A lumbar puncture revealed cerebral spinal fluid cytology that was within normal limits. Serum gram stain quickly resulted gram-negative rods. BioFire FilmArray (BioFire, Salt Lake City, UT) blood culture identification panel, a rapid polymerase chain reaction (PCR) test, resulted presumptive K. pneumoniae within 1 hour of testing. The patient was subsequently treated with intravenous (IV) ceftriaxone 2 g every 24 hours and admitted to the hospital for continued evaluation and treatment. Final blood culture speciation and sensitivities resulted heavy growth of pan-sensitive K. pneumoniae, while final urine and cerebral spinal fluid cultures remained negative.

Over the next 24 hours, the patient underwent thorough evaluation for the source of her K. pneumoniae bacteremia. This work-up included a right upper-quadrant ultrasound that was notable for a $3.9 \times 4.2 \times 4.2 \mathrm{~cm}$ lesion within the right lobe of the liver (-Fig. 1). The liver lesion was predominantly echogenic with an irregular, ill-defined peripheral rim and no internal blood flow. Due to concerns for an abscess, Infectious Disease was consulted and recommended addition of IV metronidazole $500 \mathrm{mg}$ every 8 hours to the patient's antibiotic regimen, as PLA is most often polymicrobial. The patient also underwent stool evaluation for ova and parasites since parasitic liver abscesses remain more common worldwide than PLA. The patient next underwent magnetic resonance imaging (MRI) with magnetic resonance cholangiopancreatography (MRCP) that confirmed the right hepatic lobe lesion noted by the ultrasound that was consistent with pyogenic abscess (-Fig. 2). No additional infectious focus or abnormality was noted on MRI of the abdomen and pelvis.



Fig. 2 Follow-up magnetic resonance imaging (MRI) of the abdomen/ pelvis in our patient demonstrated a $4.0 \times 4.6 \times 4.0 \mathrm{~cm}$ inferior hepatic lobe focus that was most consistent with pyogenic abscess, confirming the previous ultrasound results.

The patient underwent ultrasound and fluoroscopyguided percutaneous aspiration of the liver abscess with drain placement by Interventional Radiology. Culture confirmed monomicrobial infection with pan-sensitive $K$. pneumoniae. To rule out invasive PLA, the patient underwent echocardiogram and ophthalmologic examination, which were both normal. Ultimately, the patient was diagnosed with cryptogenic noninvasive PLA (CNPLA) secondary to $K$. pneumoniae. Prior to discharge on hospital day number 5 , the patient underwent peripherally inserted central catheter (PICC) placement due to plans for prolonged antibiotic therapy. The IV ceftrixone $2 \mathrm{~g}$ daily was continued and she was transitioned to per os (PO) metronidazole $500 \mathrm{mg}$ three times daily.

After a total of two weeks of antibiotic therapy, the patient followed-up with Infectious Disease. The IV ceftriaxone and PO metronidazole as well as the PICC line were discontinued and the patient was transitioned to PO cefixime $400 \mathrm{mg}$ daily. The plan was to continue antibiotic therapy until complete resolution of the abscess. After a total of four weeks of antibiotic therapy (two weeks IV and two weeks PO only), the patient underwent MRI confirming resolution of the abscess and the drain and antibiotics were discontinued at $\sim 15$ weeks of gestation.

For the remainder of the pregnancy, the patient was followed in the high-risk obstetric clinic. She had an uneventful antenatal course with term delivery at 37 weeks and 6 days gestation following presentation in labor with spontaneous rupture of membranes. She delivered a healthy female infant weighing 3,635 g with Apgars 8 and 9 at 1 and 5 minutes, respectively. The patient and newborn were discharged home together on postpartum day 1 .

The patient followed-up with Infectious Disease at $\sim 8$ weeks postpartum and was incidentally found to have a mild transaminitis (alanine aminotransferase [ALT], of $43 \mathrm{U} / \mathrm{L}$ compared with patient's baseline of $16 \mathrm{U} / \mathrm{L}$ ) that was expectantly managed with complete resolution at 8 months postpartum. In the 10 months since her delivery, the patient has received treatment via incision and drainage for a subcutaneous abdominal abscess (no cultures obtained) and nares 
abscess (oxacillin-resistant Staphylococcus aureus). She was also recently evaluated by her primary care physician for epigastric pain felt most likely to be secondary to acid reflux, but for which abdominal imaging was pursued given her prior history and was subsequently normal. She is scheduled for annual follow-up with Infectious Disease.

\section{Discussion}

Pyogenic liver abscesses are much more common in Eastern compared with Western countries, with $82 \%$ of cases occurring in Taiwan. ${ }^{1}$ The majority of cases are polymicrobial and occur in elderly men with diabetes mellitus who also have underlying hepatobiliary or gastrointestinal disease. Monomicrobial infection represents a minority of PLA. K. pneumoniae infection, specifically, was once a rare finding in Western cases of monomicrobial PLA; however, rates are increasing and now account for up to $40 \%$ of cases in certain areas of the United States. Interestingly, United States' cases of monomicrobial PLA secondary to K. pneumoniae have only been described in patients of Asian or Hispanic descent. This finding suggests that geographical residence as well as genetic susceptibility play a role in this poorly understood predisposition. ${ }^{2,3}$

Overall, monomicrobial CNPLA secondary to K. pneumoniae remains a rare condition worldwide with most cases clustering in Southeast Asia. In the United States, these cases differ from polymicrobial PLA as they are most often community-acquired, rarely associated with diabetes or identifiable hepatobiliary or gastrointestinal disease, and generally have a good clinical outcome if promptly diagnosed and treated. Unlike CNPLA, cryptogenic invasive PLA (CIPLA) is highly associated with distant seeding and is nearly exclusively found in Taiwan. While the pathogenesis of this finding is most likely multifactorial and related to race, environment, and underlying disease (e.g., diabetes mellitus), the precise explanation for the endemic nature of CIPLA to Taiwan remains poorly understood. ${ }^{4}$ Like CNPLA, CIPLA cases are increasingly associated with K. pneumoniae, rising from $30 \%$ in the 1980 s to $80 \%$ of cases in the 1990 s. $^{1}$

Symptoms of monomicrobial CNPLA are frequently nonspecific and routine laboratory evaluation is often nondiagnostic, particularly in cases of noninvasive disease. Rapid diagnosis is critical as associated bacteremia can progress to sepsis if left untreated, with subsequent increase in morbidity and mortality. In the pregnant patient, sepsis is additionally associated with worsening outcomes including two times greater risk for preterm delivery and five times higher rates of perinatal mortality. ${ }^{5}$ Accurate recognition necessitates a thorough work-up to include blood cultures and directed testing based on patient history and ultimately requires abdominal imaging for the diagnosis.

Antibiotic treatment of bacteremia-associated PLA is imperative and should be initiated within 60 minutes of presentation, particularly if there are concerns for sepsis. ${ }^{6}$ Most often this requires initiation of broad-spectrum antibiotic coverage prior to receiving final culture results. While gram stain and/or cytology can direct therapy, precise speciation usually requires 24 to 48 hours. However, newer PCR techniques have allowed for rapid preliminary speciation, as was the case for our patient. Available testing using these highly multiplexed PCR assays of blood, cerebral spinal fluid, and gastrointestinal and/or respiratory samples can provide preliminary results for many infections within 60 minutes.

Following initiation of antibiotics, a work-up for the source of bacteremia is necessary. In our patient, the full fever work-up was unremarkable and required additional imaging. With pulmonary and genitourinary sources ruled out, evaluation for possible intra-abdominal etiology was pursued via abdominal ultrasound. Extrapolated data from non-pregnant patients has shown abdominal ultrasound to be an effective screening tool for PLA, with greater than $85 \%$ sensitivity. ${ }^{7}$ Subsequent imaging, via CT or MRI, is often completed when PLA is suspected to both confirm ultrasound findings and assess for the underlying source of the abscess as most causes are secondary to underlying hepatobiliary disease or intra-abdominal infection. PLA often requires drainage of the abscess and necessitates prolonged antibiotic therapy.

Once cryptogenic PLA has been diagnosed and targeted treatment is initiated, it is important to evaluate for invasive infection. Septic emboli most commonly occur in the eyes (endophthalmitis) but have also been described in the lungs, brain, heart, kidneys, and colon. Necrotizing fasciitis, although rare, has also been reported. Additionally, there have been rare cases of suspected cryptogenic PLA with underlying subclinical malignancy, particularly colorectal cancer. In one study, colonoscopy detected underlying cancer in $25 \%$ of patients with cryptogenic PLA secondary to $K$. pneumonia and was most common among patients of Asian descent with diabetes mellitus. ${ }^{8}$ Therefore, colonoscopy should be performed in patients with risk factors and in those with concerning symptoms and/or imaging. Also, recurrent cryptogenic PLA may be a harbinger of underlying colorectal cancer. $^{9}$

Very few cases of perinatal PLA cases have been described, with exceedingly rare occurrence in Western countries ( - Table 1). These cases were found to be secondary to other infections; specifically Streptococcus anginosus in a case subacute cholecystitis secondary to local perforation of the gallbladder in a Canadian patient at 30 weeks of gestation ${ }^{10}$ and methicillin-resistant Staphylococcus aureus (MRSA) in an American patient at 33 weeks who was treated for multiple subcutaneous MRSA abscesses four weeks prior. ${ }^{11}$ Our case is the first to our knowledge reporting a case of cryptogenic PLA in pregnancy and is most accurately described as noninvasive. Our Filipino patient had no underlying medical conditions that would have otherwise put her at increased risk for PLA.

\section{Conclusion}

Pyogenic liver abscess remains a very rare cause of bacteremia and/or sepsis in pregnancy, particularly in Western countries. Given the diversity of the United States population 


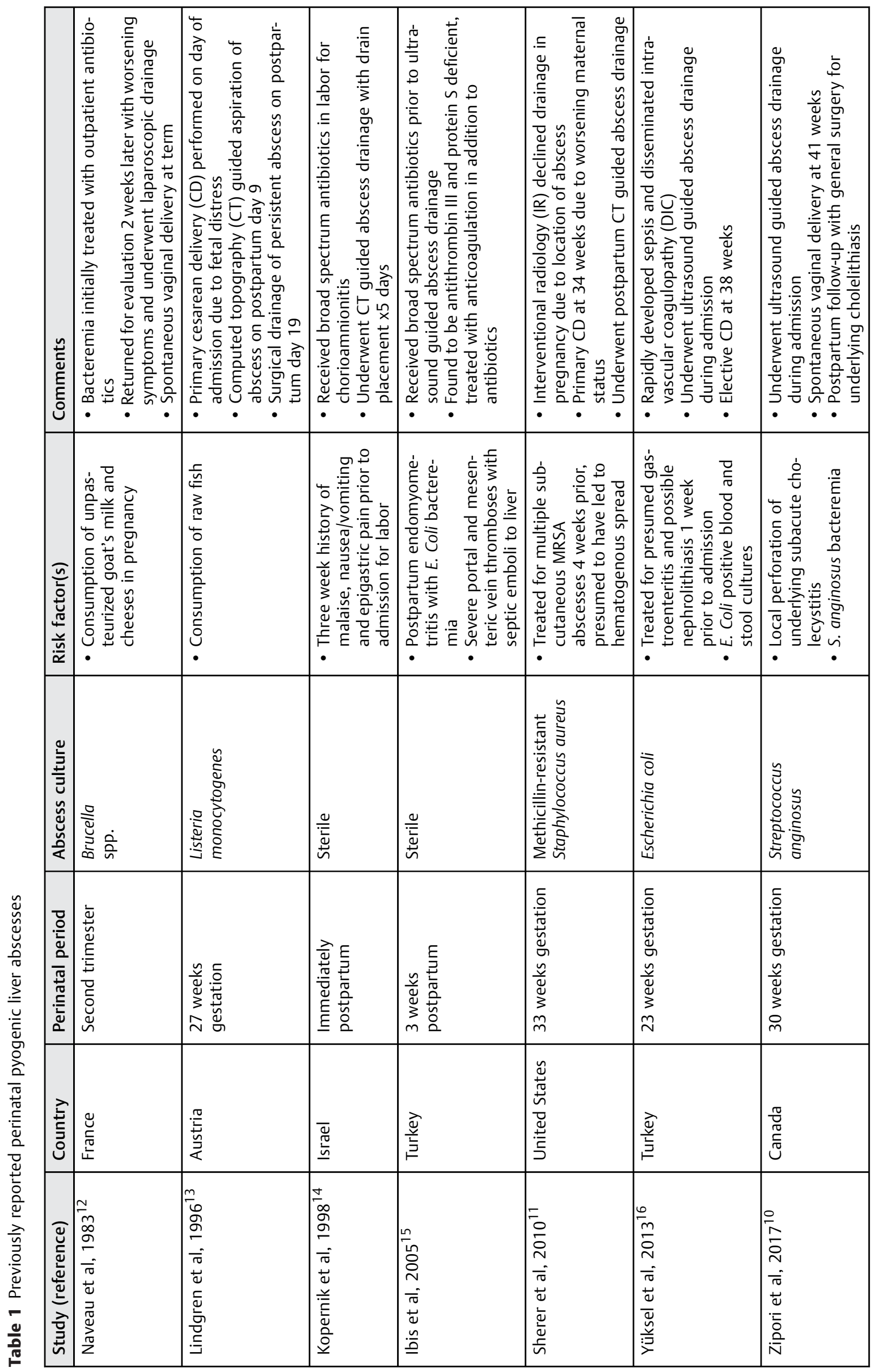


with high rates of patients of Asian and Hispanic descent, PLA should be considered in cases of sepsis where common sources have been ruled out even in the absence of underlying risk factors like diabetes, hepatobiliary disease, or gastrointestinal disease. Once recognized, targeted antibiotic therapy and drainage of the abscess will lower the maternal morbidity and mortality and decrease the subsequent risk of pregnancy complications.

The opinions expressed herein are those of the authors and do not reflect the official policy or position of Madigan Army Medical Center, the Department of the Army, the Department of Defense or the United States Government.

\section{Financial Disclosures}

None.

Précis

Perinatal pyogenic liver abscess is a rare but dangerous etiology of maternal sepsis, requiring thorough infectious work-up and prompt treatment to improve maternal-fetal outcomes.

\section{Teaching Points}

Pyogenic liver abscesses are an uncommon etiology of fever in the puerperium and are often associated with nonspecific symptoms that overlap common pregnancy complaints.

Accurate diagnosis of a pyogenic liver abscess requires thorough fever workup until a source is identified.

Astute obstetric care and a multidisciplinary approach can allow for prompt diagnosis and directed therapy, improving outcomes for both mother and fetus.

\section{Note}

This article was presented at the American College of Obstetricians and Gynecologists Annual Clinical and Scientific Meeting, Austin, Texas, April 2018.

\section{Conflict of Interest}

None.

\section{References}

1 Braiteh F, Golden MP. Cryptogenic invasive Klebsiella pneumoniae liver abscess syndrome. Int J Infect Dis 2007;11(01):16-22

2 Rahimian J, Wilson T, Oram V, Holzman RS. Pyogenic liver abscess: recent trends in etiology and mortality. Clin Infect Dis 2004;39 (11):1654-1659

3 Sink JR, Pasculle WA, Shah NB, Vergis EN. Disparate domains: cryptogenic invasive Klebsiella pneumoniae liver abscess syndrome. Am J Med 2017;130(06):673-677

4 Wang JH, Liu YC, Lee SS, et al. Primary liver abscess due to Klebsiella pneumoniae in Taiwan. Clin Infect Dis 1998;26(06): 1434-1438

5 Knowles SJ, O'Sullivan NP, Meenan AM, Hanniffy R, Robson M. Maternal sepsis incidence, aetiology and outcome for mother and fetus: a prospective study. BJOG 2015;122(05):663-671

6 Howell MD, Davis AM. Management of sepsis and septic shock. JAMA 2017;317(08):847-848

7 Lin AC-M, Yeh DY, Hsu Y-H, et al. Diagnosis of pyogenic liver abscess by abdominal ultrasonography in the emergency department. Emerg Med J 2009;26(04):273-275

8 Jeong SW, Jang JY, Lee TH, et al. Cryptogenic pyogenic liver abscess as the herald of colon cancer. J Gastroenterol Hepatol 2012;27 (02):248-255

9 Kim HY, Kim CW, Kim DR, et al. Recurrent pyogenic liver abscess as a presenting manifestation of colorectal cancer. Korean J Intern Med (Korean Assoc Intern Med) 2017;32(01):174-177

10 Zipori Y, Berger H, Colak E, Geary M. Pyogenic liver abscess with atypical microbiology during pregnancy. A case report. J Obstet Gynaecol 2017;37(06):827-828

11 Sherer DM, Dalloul M, Shah T, et al. Sonography and magnetic resonance imaging of a maternal methicillin-resistant Staphylococcus aureus liver abscess at 33 weeks' gestation. J Ultrasound Med 2010;29(06):989-992

12 Naveau S, Poitrine A, Delfraissy JF, Brivet F, Dormont J. Brucellosis hepatic abscesses and pregnancy. Gastroenterology 1983;84(06): 1643

13 Lindgren P, Pla JC, Högberg U, Tärnvik A. Listeria monocytogenesinduced liver abscess in pregnancy. Acta Obstet Gynecol Scand 1997;76(05):486-488

14 Kopernik G, Mazor M, Leiberman JR, Barki Y, Hirsch M. Pyogenic liver abscess in pregnancy. Isr J Med Sci 1988;24(4-5):245-246

15 Ibiş M, Odemiş B, Başar O, Cakal B, Beyazt F, Köklü S. Postpartum severe portal vein thrombosis and pyogenic liver abscess. J Clin Gastroenterol 2005;39(07):646

16 Yüksel B, Seven A, Kucur S, Gözükara I, Keskin N. Presentation and management of pyogenic liver abscess in a 23-week pregnant woman. Case Rep Obstet Gynaecol 2013:845215 